\section{Circulation and axonal transport in the optic nerve}

JE Morgan ${ }^{1,2}$

\begin{abstract}
Retinal ganglion cells are the output cells of the retina whose axons are under considerable metabolic stress in both health and disease states. They are highly polarised to ensure that mitochondria and enzymes involved in the generation of ATP are strategically concentrated to meet the local energy demands of the cell. In passing from the eye to the brain, axons are protected and supported by glial tissues and the blood supply of the optic nerve head is regulated to maintain the supply of oxygen and nutrients to the axons.

In spite of this, the optic nerve head remains the point at which retinal ganglion cell axons are most vulnerable to the effects of increased intraocular pressure or ischaemia.

Considerable work has been undertaken in this area to advance our understanding on the pathophysiology of axon damage and to develop new strategies for the prevention of retinal ganglion cell death.
\end{abstract}

Eye (2004) 18, 1089-1095.

doi:10.1038/sj.eye. 6701574

Keywords: optic nerve; axon; axoplasmic transport

\section{Introduction}

Retinal ganglion cells provide the axons that connect the eye with the brain. They integrate signals within the retina and are the point at which these are converted to action potentials. Since these activities are highly energy dependent and the axon path to the CNS visual centres is long, these cells are vulnerable to a range of metabolic and ischaemic insults that can act within the retina and along the course of the axon. Not surprisingly, pathological conditions such as glaucoma or hereditary optic neuropathies that cause selective retinal ganglion cell death result in profound loss of vision.

The pathway taken by optic nerve axons as they cover the $5 \mathrm{~cm}$ between the eye and the visual centres is stressful for the cell. On leaving the eye, axons turn through $90^{\circ}$ to enter the optic nerve head and then traverse the lamina cribrosa to enter the retrobulbar optic nerve. The route taken by individual axons can place them at increased risk of damage. Clinical studies based on high contrast red-free photographs of the retinal nerve suggest a high degree of topographic organisation for axon bundles based on the orientation of retinal astrocytes that form the visible striations in the retinal nerve fibre layer. ${ }^{1-3}$ By contrast, studies of the individual axon pathways suggest a lower degree of topographic precision. In the transverse plane of the retina, axons pass between bundles; ${ }^{4,5}$ in the vertical plane axons from cells at the same retinal eccentricity can pass at different levels within the retinal nerve fibre layer. ${ }^{4}$ The organisation within the retrobulbar part of the nerve is relatively straightforward, in that axons from peripheral retina pass in the peripheral part of the nerve, while those from more central retinal loci pass through central aspects of the nerve. ${ }^{6}$ Even so, the precision with which parts of the optic nerve are retinotopically demarcated is coarse, with considerable overlap between the regions. ${ }^{6}$ To achieve this order it has been suggested that more centrally arising axons pass through overlying axons so that they lie in the more superficial part of the nerve. ${ }^{3}$ Tracing studies of individual axon paths suggest that this may not be the case and that some axons undergo a complex partial decussation at the margin of the optic nerve head, in which axons from peripheral and central retinal ganglion cells mingle to adopt the correct location within the retrobulbar optic nerve. ${ }^{4,7,8}$ In vitro tracing of individual axon paths in the human optic nerve head has shown that some axons do not take a direct course through the lamina cribrosa. ${ }^{9}$
${ }^{1}$ Department of Ophthalmology University Hospital of Wales Heath Park Cardiff, UK

${ }^{2}$ School of Optometry and Vision Sciences

Edward VII Avenue

Cardiff, UK

Correspondence: JE Morgan School of Optometry and

Vision Sciences

Edward VII Avenue

Cardiff CF10 3NB, UK

Tel: + 442920743222

Fax: + 442920874859

E-mail: morganje3@

cardiff.ac.uk

Received: 4 September 2003

Accepted: 4 September 2003 
Rather than taking a direct path through the optic nerve head and lamina cribrosa, axons can deviate from these paths at the margin of the optic nerve head and in some cases pass between the plates of the lamina cribrosa.

The optic nerve head provides a supportive environment for retinal ganglion cell axons. Almost 50\% of the cells in the optic nerve head comprise glia.

Astroctyes form a major part of this population and form glial tubes in the prelaminar part of the optic nerve through which bundles of axons run to enter the optic nerve. ${ }^{10,11}$ Astrocytes are critical for preserving the correct cellular environment for retinal ganglion cell axons. They are connected by gap junction $\mathrm{s}^{12}$ that allow them to act as a syncytium to buffer changes in the extracellular environment of the axon. At the posterior part of the scleral laminar, axons are wrapped by oligodendrocytes as they enter the retrolaminar part of the optic nerve. Myelination confers a great advantage for the cell since it allows saltatory conduction of action potentials that reduces the energy demand for the cell.

\section{Axon dimensions}

Consideration of the relative dimensions of the axon and retinal ganglion cells indicates the magnitude of the metabolic demands that are placed on the retinal ganglion cell axons. While the cell soma is the principal site for energy production and protein synthesis, there is evidence that the axon in its own right, has, to supplement some of these functions. If the median value for the soma diameter for larger retinal ganglion cells (mostly parasol cells) is taken as $19 \mu \mathrm{m}$, mean the axon diameter as $0.95 \mu \mathrm{m}$ and axon length as $5 \mathrm{~cm}$ based on published data, ${ }^{13,14}$ it can be shown that the axon volume would comprise $80 \%$ of the total cell volume. If comparison is made on the basis of cell area (which provides a better estimate of metabolic demand), the disparity is more marked with the axon comprising 93\% of the cell area. In view of this, it is not surprising that the optic nerve head is specialised to provide a supportive environment for the axon. This can be seen in two ways, firstly, in the provision of mitochondria and metabolic support to satisfy the energy demands of the axon and secondly, in terms of the blood supply to the optic nerve head to satisfy these demands.

\section{Modifications for metabolic demand}

Mitochondria are the site for the oxidative generation of ATP and tend to accumulate in regions with high energy demand. In the retina, it seems likely that collections of mitochondria contribute to the varicosities that can be seen within the healthy retinal ganglion cell axon. ${ }^{5}$ Immunohistochemical studies of the normal human optic nerve head show high concentrations of mitochondria as evidenced by staining for the mitochondrial enzyme cytochrome oxidase, ${ }^{15,16}$ which are concentrated around sodium channels required for the conduction of action potentials (Figure 1). Recent evidence suggests that the apparent dilatation of retinal ganglion cell axons within the lamina cribrosa that were thought to result from axonal compression by the cribrosal plates may reflect the accumulation of mitochondria to support the high
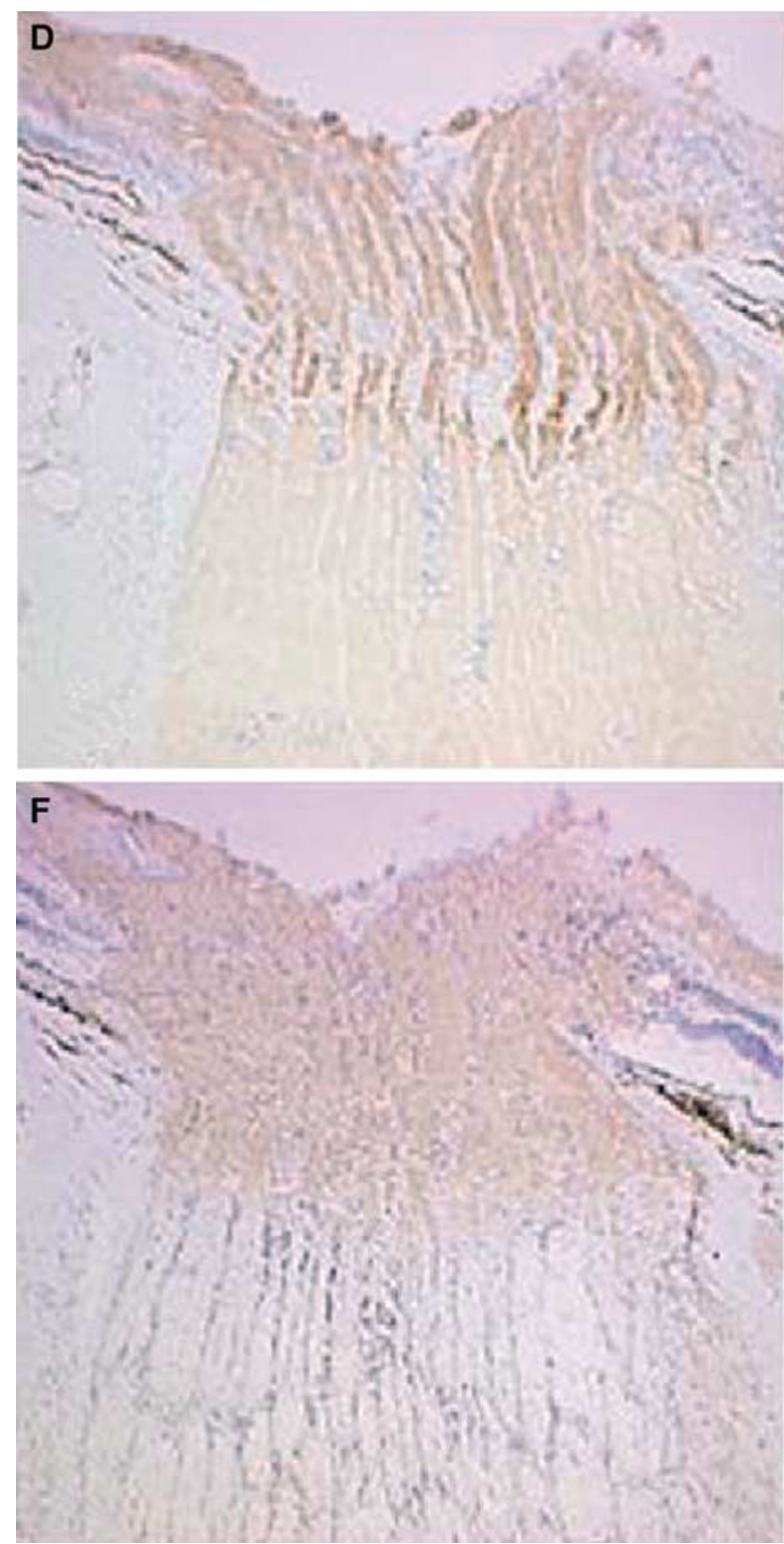

Figure 1 Cryostat sections from a normal optic nerve head. (D) (upper): Immunohistochemical stain for the COX enzyme indicating concentration of mitochondria within the optic nerve head. (F) (lower): Immunohistochemical stain for the voltagesensitive sodium channel showing a similar distribution with the COX distribution. From Barron et al ${ }^{15}$ (adapted from Figure 1). Source: Ref 15 British Journal of Ophthalmology. 
energy demands of axons in this part of the optic nerve. ${ }^{15}$ Mitochondria do not appear to occupy a fixed location within the axon since they can move to regions where the demand is high. During development, for example, mitochondria move to regions where the axonal growth cone is expanding. ${ }^{17}$ When the growth is complete, mitochondria then redistribute throughout the neuron. ${ }^{18}$ The critical importance of mitochondria to the survival of retinal ganglion cells is emphasised in diseases such as Lebers optic neuropathy or autosomal dominant optic neuropathy, ${ }^{19,20}$ where mitochondrial dysfunction results in retinal ganglion cell death and profound vision loss.

\section{Blood supply - tailored to demands}

Detailed descriptions of the optic nerve head blood supply have been given elsewhere $\mathrm{e}^{21,22}$ and so will only be dealt with briefly here. In keeping with the metabolic demands of the axons, the optic nerve head has a rich plexus of blood vessels and fine capillaries. These are derived from branches of the ophthalmic artery that gives off the central retinal artery as it enters the orbit and two posterior ciliary arteries that travel on either side of the retrobulbar optic nerve to penetrate the sclera on either side of the optic nerve. The central retinal artery enters the optic nerve on its ventral aspect approximately $10 \mathrm{~mm}$ behind the eye. Within the sclera, the posterior ciliary arteries branch to form a ring of vessels around the optic nerve head, the short posterior ciliary vessels, which then supply axons in the prelaminar and laminar parts of the optic nerve head. They also give rise to the long posterior ciliary arteries that pass forward to supply structures in the anterior segment. It is important to note that the central retinal artery does not supply the anterior aspect of the optic nerve head though it does supply peripapillary retina. The short posterior ciliary vessels form a variable anastomosis around the anterior optic nerve head, which has been reported as complete in up to $77 \%$ of cases studied, with others showing areas of focal narrowing or incomplete anastomosis. ${ }^{23}$

Deficiencies in this anastomosis are thought to underlie the clinical presentation of conditions such as anterior ischaemic optic neuropathy in which altitudinal visual field defects arise because of the existence of watershed zones within the optic nerve head circulation. ${ }^{22}$ Further branches to the optic nerve head also come from choroidal arterioles.

\section{Autoregulation}

Maintenance of a stable oxygen supply is essential to support the metabolic demands of optic nerve head axons; this has to be carried out in spite of variations in intraocular and systemic blood pressure. Clinical estimates of optic nerve head blood flow by laser Doppler techniques have demonstrated a considerable ability for the optic nerve head vasculature to maintain perfusion over a range of intraocular pressures. ${ }^{24}$ However, the dynamic range for this autoregulation is limited, with perfusion reducing above $45 \mathrm{mmHg}$ intraocular pressure (Figure 2). Interestingly, these measurements suggest that the ability to autoregulate may be deficient in some individuals and that it may also vary regionally within a single optic nerve head. More direct measurements of autoregulation by in vivo measurements of $\mathrm{O}_{2}$ levels within the optic nerve of miniature pigs (which have many features in common with the human optic nerve head) show that these remain constant during periods of hypoxia or induced
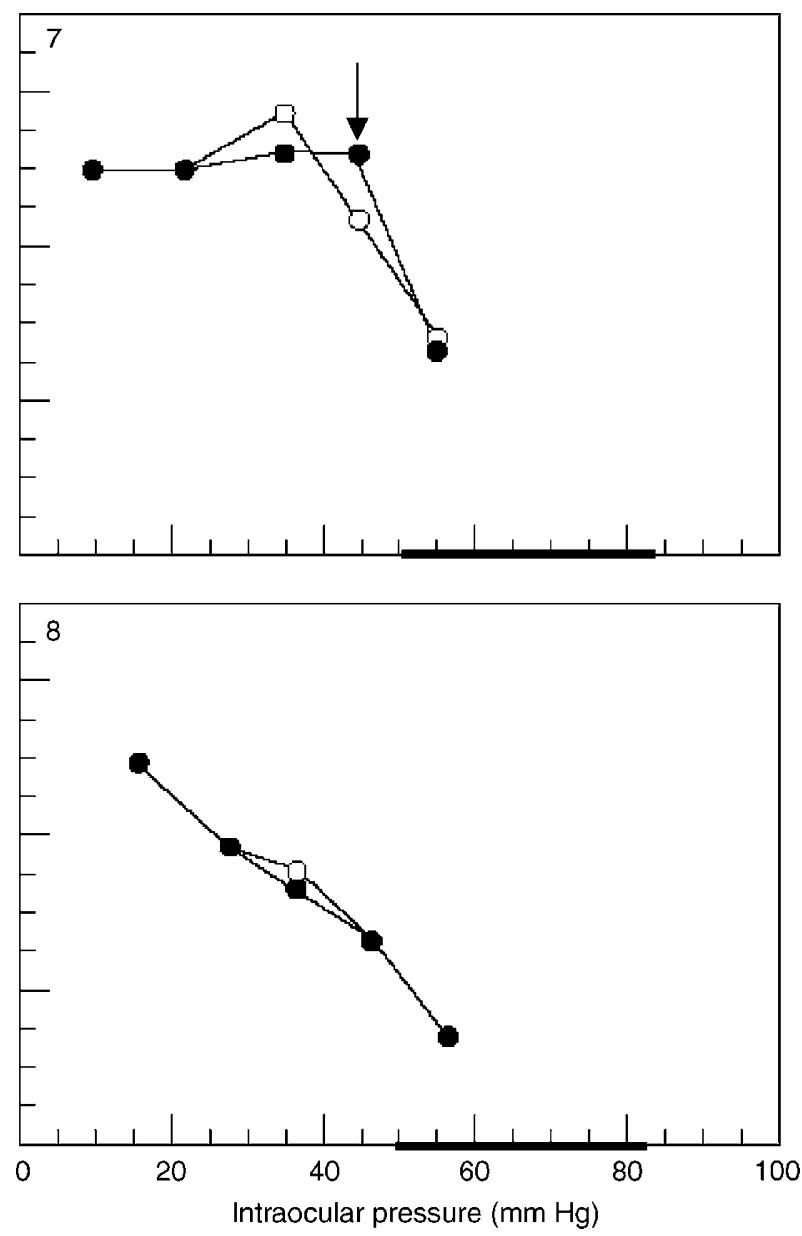

Figure 2 Percentage blood flow relative to baseline ( $y$-axis) for two subjects, plotted against intraocular pressure. Clear circles, immediately after pressure elevation. Filled circles, $60 \mathrm{~min}$ after pressure elevation. Upper plot: good autoregulation in maintained until just over $40 \mathrm{mmHg}$ intraocular pressure (indicated by arrow). Lower plot: autoregulation is not effective for intraocular pressures over $20 \mathrm{mmHg}$. From Pillunat et $a l^{24}$ (adapted from Figure 3). Source: Ref 24 Experimental Eye Research. 
systemic hypotension. ${ }^{25}$ The autoregulation provides a finite range of preserved perfusion which, if exceeded, will result in optic nerve head ischaemia. Profound nocturnal hypotension and optic nerve head under perfusion following systemic shock have both been associated with the development of glaucomatous cupping. ${ }^{26,27}$

Several groups have investigated the factors that may play a role in this autoregulation. Since there are no precapillary sphincters within the capillary bed of the optic nerve head, interest has been focused on the capillary pericytes that have contractile properties that allow them to vary the resistance within the capillary bed and thereby adjust the resistance to perfusion. ${ }^{28,29}$ In vitro studies have shown that pericytes within the optic nerve head will respond by constricting when the $\mathrm{O}_{2}$ is reduced or the $\mathrm{pH}$ is increased (in turn reflecting the level of $\mathrm{pCO}_{2}$ ).

Adenosine can be particularly potent by relaxing pericytes and ensuring increased blood flow in areas of high metabolic demand (and therefore high use of ATP). ${ }^{30}$ All these mechanisms act in concert to maintain appropriate levels of $\mathrm{O}_{2}, \mathrm{CO}_{2}, \mathrm{~K}^{+}$and $\mathrm{pH}$ within the optic nerve head. It seems likely that systemic factors, for example angiotensin II, may also act to moderate the response to $\mathrm{CO}_{2}{ }^{31}$

Endothelial cells also play an important role in controlling vascular tone within the optic nerve head. Early experiments covering the role of endothelial cells in the regulation of vascular tone revealed the importance of nitric oxide in increasing blood flow through the capillary bed. Abnormalities in nitric oxide synthesis have been reported in diseases such as glaucoma, diabetes, and retinopathy of prematurity where compromised optic nerve head blood flow may be involved in retinal pathophysiology. ${ }^{32}$ Endothelins also play an important role in the regulation of endothelial function and in the control of vascular tone. Chronic administration of endothelin 1 can cause marked vasoconstriction in the optic nerve head circulation, resulting in retinal ganglion cell death in animal models. ${ }^{33,34}$ Transient increases in plasma endothelin may be associated with ischaemic optic neuropathy (case report) ${ }^{35}$ Endothelin receptors have been found on optic nerve head astrocytes ${ }^{36}$ that regulate astrocyte

proliferation in disease states and directly influence axon function. Elevated endothelin levels can also decrease the anterograde transport of mitochondria in animal models ${ }^{37}$ thereby compromising local supplies of ATP.

\section{Axoplasmic transport}

In addition to its primary function in conducting action potentials, the axon allows the cell body to communicate metabolically with its terminal neuronal targets in the lateral geniculate nucleus and the superior colliculus.
These processes are instrumental in determining neuronal survival in development and disease. This communication is achieved by the transport of molecules, vesicles, and organelles in both an anterograde (also known as orthograde; away from the cell body, toward the brain) and retrograde (toward the cell body, away from the brain) directions. Disruption in this transport process, either by focal damage to the optic nerve or by damage to the cell population contacted by the axon terminal, can result in retinal ganglion cell death. In experimental glaucoma, for example, there is evidence that a reduction in the levels of BDNF (which is retrogradely transported to the retinal ganglion cell body) is associated with increased retinal ganglion cell death, ${ }^{38}$ the exogenous administration of BDNF can protect these cells against such damage. ${ }^{39}$

\section{Anterograde transport}

Anterograde transport can be divided into the following processes:

(i) Fast anterograde transport occurs at a rate of $50-400 \mathrm{~mm} /$ day and is related to the transport of synaptic vesicles proteins, kinesins, and enzymes involved in the metabolism of neurotransmitters.

(ii) Slow anterograde transport is given over to the transport of neuronally synthesised proteins that include cytoskeletal components, polymers, and protein complexes that are to be delivered to the axon and its terminal regions. It is divided into two types: ${ }^{40}$ slow axonal transport component A (SCa) $(0.3-3 \mathrm{~mm} /$ day), which is concerned with the transport of neurofilament triplet proteins such as tubulin and spectrin (as well as tau proteins). The other system, slow axonal transport component B $(\mathrm{SCb})$, is slightly faster $(2-8 \mathrm{~mm} /$ day $)$ and is concerned with the transport of microfilaments, actin, and supramolecular complexes of cytosolic matrix proteins. There has been considerable discussion concerning the mechanisms of slow axonal transport. Studies in which fluorescent tags are placed on tubulin have allowed delineation of the microtubular organisation of the axon suggest that neurofilaments may move intermittently at fast speeds (up to $2 \mu \mathrm{m} / \mathrm{s}$ ) interspersed with a period of no motion. ${ }^{41}$ The overall result is of apparent slow axonal transport. A curious feature of this system is the wide variation in transport speeds for different molecules. It is possible that this reflects the affinity for any given molecule for the transport system. High-affinity binding would result in more continuous (and therefore faster) transport compared with low-affinity binding in which transport is more intermittent (and therefore, slower).

The molecular motor for anterograde transport is provided by kinesin molecules that are part of a family of 
specialised motor proteins. Kinesins have a conserved motor domain that hydrolyses ATP to generate movement along the microtubules running down the axon. The kinesins are synthesised in the cell body and stored in a soluble form in the cytoplasm. The kinesin motor is activated on binding to the 'cargo' molecule (the molecule or cellular component to be transferred along the axon), which then tracks along the axonal microtubules to the axon tip. The microtubules themselves are highly polarised structures composed of alpha and beta tubulin. By convention, they have a stable 'minus' end close to the cell body and a 'positive' end close to the axon tip, which is usually more unstable and is associated with the growing end of the axon. Several different kinesin motors exist that may explain the different rates of transport; the activity of various kinesin subtypes can be regulated independently to ensure that cargoes are delivered as required. ${ }^{42}$ Axonal myosins ${ }^{43}$ are involved in the delivery of the cargo molecule at axon terminals and may also modulate other aspects of fast anterograde axonal transport.

\section{Retrograde transport}

Retrograde transport is classed as fast (200-400 mm/day) and is concerned with the movement of endosomes and
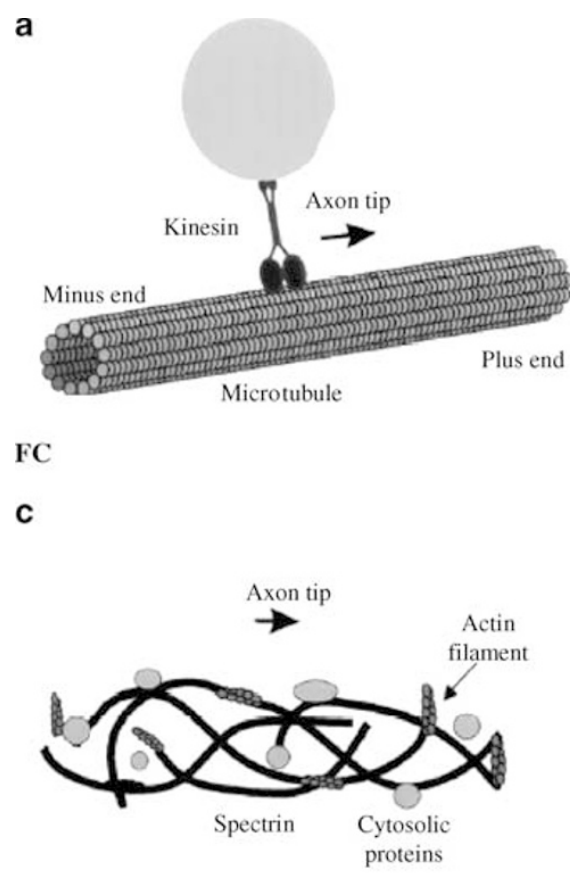

$\mathrm{SCb}$ lysosomes containing internalised membrane receptors and neurotrophins towards the cell body. It uses dynein as the molecular motor (Figure 3). ${ }^{4,45}$ Dynein is first synthesised in the cell body, transported to the axon tip (the 'positive' end) by both fast and slow anterograde transport systems, ${ }^{46}$ and then activated ${ }^{47}$ to commence retrograde transport toward the cell body. The structure of the dynein has been well characterised. One part of the molecule has a microtubule binding site that is connected to a collection of subunits for binding to molecules that are to be transported. ${ }^{45}$ The composition of the subcomponents of the dynein molecule varies and has given rise to the existence of two pools of dynein within the cytoplasm that may underlie some of the differences in the transport rates for particular molecules. For the slower retrograde transport, the dynein is associated with actin-spectrin meshwork within the axon, rather than the microtubules.

\section{Intra-axonal synthesis}

The rate of slow axonal transport suggests that it would not be suitable for transporting molecules over long distances; for some axons it could take several years for molecules moving in this system to reach the axon tip. One solution to this problem comes from evidence that

b
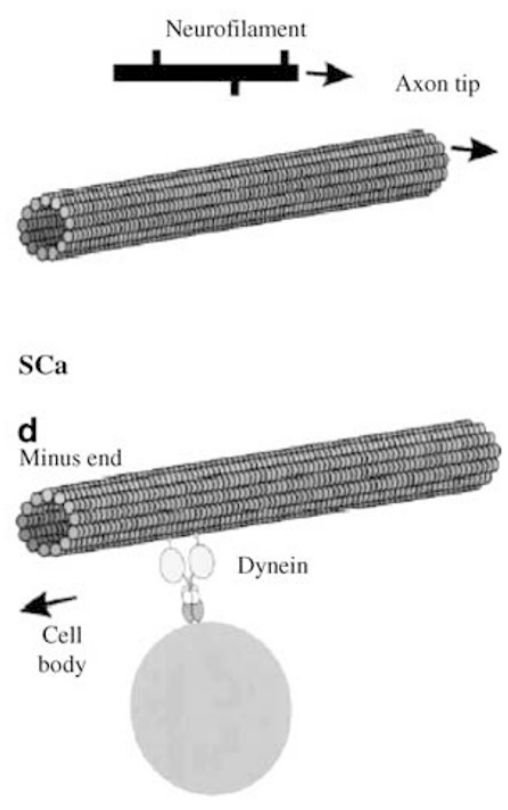

Retrograde

Figure 3 (a) Relationship of the kinesin family of molecules with the microtubules within the axons for fast anterograde axoplasmic transport. (b) and (c) are the slow component using the microtubules or actin-spectrin system, respectively. (d) Retrograde transport system uses the dynein molecular motor acting on the axon microtubules. From Susalka and Pfister. ${ }^{45}$ Source: Ref 45 Journal of Neurocytology. 


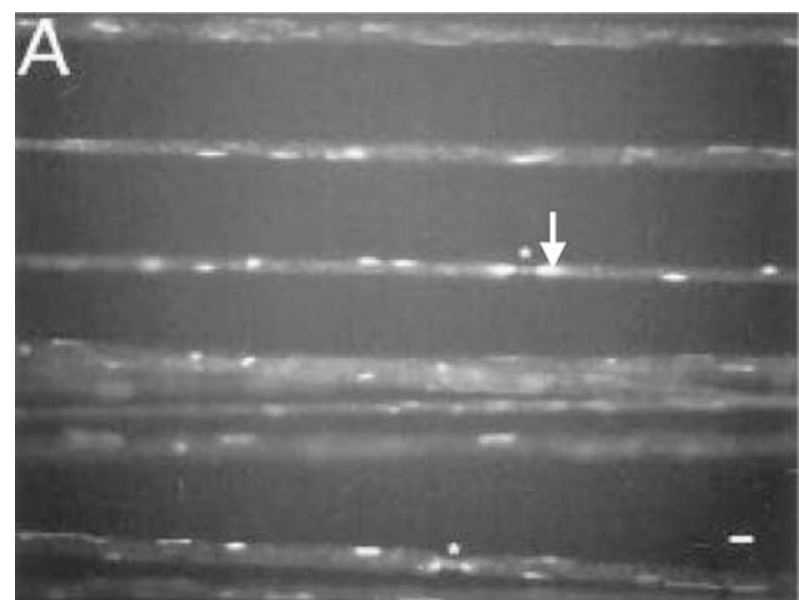

Figure 4 Periaxoplasmic plaques stained with the high-affinity nucleic acid binding dye YOYO-1. Arrow indicates typical area of hyperflourescence corresponding to the location of ribosomal plaques for the intra-axonal protein synthesis. From Koenig et al. ${ }^{45}$ Source: Ref 49 Journal of Neuroscience.

the axon itself is also an important source of protein synthesis. ${ }^{48}$ Periaxonal ribosomal plaques have recently been described, which suggest that the axon may be the site of intrinsic (and local) protein synthesis ${ }^{49}$ (Figure 4).

\section{Conclusion}

The optic nerve has proved to be an excellent model for understanding factors that regulate axon function and ensure that the neuronal energy demands are met. Although much progress has been made, therapeutic options to treat conditions that compromise axon function and result in retinal ganglion cell death remain limited. A greater understanding of the factors that control axoplasmic transport and regulate optic nerve head blood flow and neuronal mitochondrial function is essential if we are to formulate new therapeutic strategies for the preservation of vision in many common diseases of the optic nerve.

\section{References}

1 Airaksinen P, Nieminen $\mathrm{H}$. Retinal nerve fiber layer photography in glaucoma. Am J Ophthalmol 1985; 92: 877-879.

2 Quigley H, Addicks E. Quantitative studies of retinal nerve fiber layer defects. Arch Ophthalmol 1982; 100: 807-814.

3 Radius R, Anderson D. The course of axons through the retina and optic nerve head. Arch Ophthalmol 1979; 97: 1154-1158.

4 Fitzgibbon T, Taylor S. Retinotopy of the human retinal nerve fibre layer and optic nerve head. J Comp Neurol 1996; 375: 238-251.

5 Wang L, Dong J, Cull G, Fortune B, Cioffi GA. Varicosities of intraretinal ganglion cell axons in human and nonhuman primates. Invest Ophthalmol Vis Sci 2003; 44(1): 2-9.
6 Naito J. Retinogeniculate fibers in the monkey optic nerve: a demonstration of the fiber pathways by retrograde axonal transport of WGA-HRP. J Comp Neurol 1989; 284: 174-186.

7 Ogden T. Nerve fiber layer of the macaque retina: retinotopic organization. Invest Ophthalmol Vis Sci 1983; 24: 85-98.

8 Fitzgibbon T. The human fetal retinal nerve fiber layer and optic nerve head: a DiI and DiA tracing study. Vis Neurosci 1997; 14: 433-447.

9 Morgan JE, Jeffery G, Foss AJ. Axon deviation in the human lamina cribrosa. Br J Ophthalmol 1998; 82(6): 680-683.

10 Hernandez M, Neufeld A. The extracellular matrix of the optic nerve. In: Drance S, Neufeld A, van Buskirk E (eds). Pharmacology of the Glaucomas. Williams and Wilkins: Baltimore, 1992, pp 236-252.

11 Hernandez MR. The optic nerve head in glaucoma: role of astrocytes in tissue remodeling. Prog Retin Eye Res 2000; 19(3): 297-321.

12 Quigley H. Gap junctions between optic nerve head astrocytes. Invest Ophthalmol Vis Sci 1977; 16: 582-585.

13 Quigley HA, Dunkelberger G, Green W. Chronic human glaucoma causing selectively greater loss of large optic nerve fibres. Ophthalmol 1988; 95(3): 357-363.

14 Curcio C, Allen K. Topography of ganglion cells in human retina. J Comp Neurol 1990; 300: 5-25.

15 Barron MJ, Griffiths P, Turnbull DM, Bates D, Nichols P. The distributions of mitochondria and sodium channels reflect the specific energy requirements and conduction properties of the human optic nerve head. Br J Ophthalmol 2004; 88(2): 286-290.

16 Andrews R, Griffiths P, Johnson M, Turnbull D. Histochemical localisation of mitochondrial enzyme activity in human otpic nerve and retina. Br J Ophthalmol 1999; 83: 231-235.

17 Chada SR, Hollenbeck PJ. Mitochondrial movement and positioning in axons: the role of growth factor signaling. J Exp Biol 2003; 206(Part 12): 1985-1992.

18 Morris RL, Hollenbeck PJ. The regulation of bidirectional mitochondrial transport is coordinated with axonal outgrowth. J Cell Sci 1993; 104(Part 3): 917-927.

19 Votruba M, Moore AT, Bhattacharya SS. Clinical features, molecular genetics, and pathophysiology of dominant optic atrophy. J Med Genet 1998; 35(10): 793-800.

20 Howell N. LHON and other optic nerve atrophies: the mitochondrial connection. Dev Ophthalmol 2003; 37: 94-108.

21 Hayreh S. Progress in the understanding of the vascular etiology of glaucoma. Curr Opin Ophthalmol 1994; 5(11): 26-35.

22 Hayreh S. Blood supply of the optic nerve head and its role in optic atrophy, glaucoma, and oedema of the optic disc. $\mathrm{Br}$ J Ophthalmol 1969; 53: 721-748.

23 Olver J, Spalton D, McCartney A. Quantitative morphology of human retrolaminar optic nerve vasculature. Invest Ophthalmol Vis Sci 1994; 35: 3858-3866.

24 Pillunat LE, Anderson DR, Knighton RW, Joos KM, Feuer WJ. Autoregulation of human optic nerve head circulation in response to increased intraocular pressure. Exp Eye Res 1997; 64(5): 737-744.

25 Bouzas E, Donati G, Pournaras C. Distribution and regulation of the optic nerve head tissue $\mathrm{PO}_{2}$. Surv Ophthalmol 1997; 42: S27-S34.

26 Drance SM, Morgan RW, Sweeney VP. Shock-induced optic neuropathy: a cause of nonprogressive glaucoma. $N$ Engl J Med 1973; 288(8): 392-395. 
27 Graham SL, Drance SM. Nocturnal hypotension: role in glaucoma progression. Surv Ophthalmol 1999; 43(Suppl 1): S10-S16.

28 Anderson DR. Glaucoma, capillaries and pericytes. 1. Blood flow regulation. Ophthalmologica 1996; 210(5): 257-262.

29 Anderson DR, Davis EB. Glaucoma, capillaries and pericytes. 5. Preliminary evidence that carbon dioxide relaxes pericyte contractile tone. Ophthalmologica 1996; 210(5): 280-284.

30 Matsugi T, Chen Q, Anderson DR. Adenosine-induced relaxation of cultured bovine retinal pericytes. Invest Ophthalmol Vis Sci 1997; 38(13): 2695-2701.

31 Matsugi T, Chen Q, Anderson DR. Suppression of $\mathrm{CO}_{2-}$ induced relaxation of bovine retinal pericytes by angiotensin II. Invest Ophthalmol Vis Sci 1997; 38(3): 652-657.

32 Schmetterer L, Polak K. Role of nitric oxide in the control of ocular blood flow. Prog Retin Eye Res 2001; 20(6): 823-847.

33 Cioffi G, Orgul S, Onda E, Bacon D, Van Buskirk E. An in vivo model of chronic optic nerve ischemia: the dosedependent effects of endothelin- 1 on the optic nerve microvasculature. Curr Eye Res 1995; 14: 1147-1153.

34 Chauhan BC, LeVatte TL, Jollimore CA, Yu PK, Reitsamer HA, Kelly ME et al. Model of endothelin-1-induced chronic optic neuropathy in rat. Invest Ophthalmol Vis Sci 2004; 45(1): 144-152.

35 Haufschild T, Shaw SG, Kaiser HJ, Flammer J. Transient raise of endothelin-1 plasma level and reduction of ocular blood flow in a patient with optic neuritis. Ophthalmologica 2003; 217(6): 451-453.

36 Prasanna G, Krishnamoorthy R, Clark AF, Wordinger RJ, Yorio T. Human optic nerve head astrocytes as a target for endothelin-1. Invest Ophthalmol Vis Sci 2002; 43(8): 2704-2713.

37 Stokely ME, Brady ST, Yorio T. Effects of endothelin-1 on components of anterograde axonal transport in optic nerve. Invest Ophthalmol Vis Sci 2002; 43(10): 3223-3230.
38 Pease ME, McKinnon SJ, Quigley HA, Kerrigan-Baumrind LA, Zack DJ. Obstructed axonal transport of BDNF and its receptor TrkB in experimental glaucoma. Invest Ophthalmol Vis Sci 2000; 41(3): 764-774.

39 Chen $\mathrm{H}$, Weber AJ. BDNF enhances retinal ganglion cell survival in cats with optic nerve damage. Invest Ophthalmol Vis Sci 2001; 42(5): 966-974.

40 Black MM, Lasek RJ. Slow components of axonal transport: two cytoskeletal networks. J Cell Biol 1980; 86(2): 616-623.

41 Wang L, Ho CL, Sun D, Liem RK, Brown A. Rapid movement of axonal neurofilaments interrupted by prolonged pauses. Nat Cell Biol 2000; 2(3): 137-141.

42 Muresan V. One axon, many kinesins: what's the logic? J Neurocytol 2000; 29(11-12): 799-818.

43 Bridgman PC, Elkin LL. Axonal myosins. J Neurocytol 2000; 29(11-12): 831-841.

44 Schnapp BJ, Reese TS. Dynein is the motor for retrograde axonal transport of organelles. Proc Natl Acad Sci USA 1989; 86(5): 1548-1552.

45 Susalka SJ, Pfister KK. Cytoplasmic dynein subunit heterogeneity: implications for axonal transport. J Neurocytol 2000; 29(11-12): 819-829.

46 Dillman III JF, Dabney LP, Pfister KK. Cytoplasmic dynein is associated with slow axonal transport. Proc Natl Acad Sci USA 1996; 93(1): 141-144

47 Dillman III JF, Pfister KK. Differential phosphorylation in vivo of cytoplasmic dynein associated with anterogradely moving organelles. J Cell Biol 1994; 127(6 Part 1): 1671-1681.

48 Giuditta A, Kaplan BB, van Minnen J, Alvarez J, Koenig E. Axonal and presynaptic protein synthesis: new insights into the biology of the neuron. Trends Neurosci 2002; 25(8): 400-404.

49 Koenig E, Martin R, Titmus M, Sotelo-Silveira JR. Cryptic peripheral ribosomal domains distributed intermittently along mammalian myelinated axons. J Neurosci 2000; 20(22): 8390-8400. 